\title{
An Evaluation of Right-Sided Symptom Onset as a Predictor of Poor Parkinson's Disease Prognosis
}

\section{Abstract \\ Objective}

Asuman Orhan Varoğlu ${ }^{1}$, Adem Aydin ${ }^{2}$

1. Neurology, Istanbul Medeniyet University, Istanbul, TUR 2. Neurology, Kastamonu State Hospital, Kastamonu, TUR

Corresponding author: Asuman Orhan Varoğlu, asumanorhan69@gmail.com

The current study aimed to measure and compare neurological disability in Parkinson's disease (PD) patients with right-sided symptom onset with that in PD patients with left-sided symptom onset, using the measurements taken at their first and last visits, to determine if right-sided symptom onset was predictive of a poor PD prognosis.

\section{Methods}

One hundred and forty-three PD patients were included in the study. The Unified Parkinson's Disease Rating Scale (UPDRS) and the Hoehn and Yahr Scale were used to measure neurological disability in patients at the first and last visits. The scores for the neurological disability of patients at the first and last visits were compared retrospectively relative to disease onset.

\section{Results}

Seventy-six PD patients had right-sided symptom onset (53\%), and 67 patients had left-sided symptom onset $(47 \%)(p=<0.001)$. The differences between the scores at the first and last visits, measured using the UPDRS and the Hoehn and Yahr Scale, were higher for PD patients with right-sided symptom onset than patients with left-sided symptom onset ( $p=<0.001, p=<0.002$, respectively). Similarly, the UPDRS Part II and Part III values, used to evaluate motor function, were higher in PD patients with right-sided symptom onset as compared to those with left-sided symptom onset at the first and last visits $(p=<0.001)$.

\section{Conclusion}

Right-sided symptom onset was predictive of a poor prognosis in PD patients at follow-up.

Categories: Internal Medicine, Neurology, Psychology

Keywords: parkinson's disease, prognosis, clinical symptoms, asymmetry

Review began 02/14/2021 Review ended 02/16/2021 Published 02/22/2021

\section{() Copyright 2021}

Orhan Varoğlu et al. This is an open access article distributed under the terms of the Creative Commons Attribution License CC-BY 4.0., which permits unrestricted use, distribution, and reproduction in any medium, provided the original author and source are credited.

\section{Introduction}

Parkinson's disease (PD) is a chronic neurodegenerative disease that occurs due to the progressive loss of dopaminergic neurons in the substantia nigra. The clinical manifestations of PD often begin asymmetrically. In the absence of motor asymmetry in the clinical symptoms of such patients, the differential diagnosis of the condition should include other diseases [1]. After the loss of neurons (50\%-70\%) in the substantia nigra, clinical symptoms and signs that are contralateral to the affected substantia nigra are observed [2].

Reasons for asymmetric motor symptoms in PD have been speculated in the literature [3-4]. Toxic or metabolic intoxications, environmental exposure, or genetic or structural changes are thought to cause predominantly unilateral motor manifestations. The number of dopaminergic neuron cells in the right and left hemispheres of healthy individuals has been reported to differ by $30 \%$. Conversely, such a difference was not reported elsewhere [3-6]. Regardless, the difference in the distribution of dopaminergic neurons between the hemispheres may be insufficient in explaining motor predominance. Consequently, most research has only randomly described motor predominance in patients with PD [1].

Consideration has been given to the prognosis of PD patients, most of which have primarily related to biomarkers or clinical data. Asymmetric brain immune modulation between the hemispheres has been identified in many studies [7-9]. In addition, peripheral asymmetric immune function in various diseases has been demonstrated [10-12]. The first clinical study to demonstrate central immune asymmetry in multiple sclerosis patients was conducted by the authors of the current research [13].

Thus, the current study aimed to measure and compare neurological disability in PD patients with rightsided symptom onset, measured at their first and last visits, with PD patients with left-sided symptom onset, to determine if right-sided clinical signs at onset were predictive of a poor PD prognosis. 


\section{Materials And Methods}

This study was retrospectively conducted at Istanbul Medeniyet University, Goztepe Research and Educational Hospital, Istanbul, Turkey, and the Department of Neurology, Kastamonu State Hospital, Kastamonu, Turkey. One hundred and forty-three patients with idiopathic PD, aged 40-90 years, were included. Patients with Parkinson-plus syndromes, secondary parkinsonism, destructive lesions caused by other brain disorders, and other neurological diseases were excluded. The outpatient records of patients who presented between 2015 and 2020 were included.

The United Parkinson's Disease Rating Scale (UPDRS) was used to evaluate the clinical status of the patients [14]. The scores obtained using the UPDRS and Hoehn and Yahr Scale for PD patients with right-sided symptom onset, measured at their first and last visits, were compared with those of patients with left-sided symptom onset. Thus, information about disease prognosis was evaluated according to the side on which the clinical signs first manifested. The first and last presentations of all patients were evaluated by the authors themselves. The UPDRS and Hoehn and Yahr Scale scores were assessed in relation to the age, gender, disease duration, and hand dominance of the patients.

Institutional ethics committee approval was obtained from the Karabuk University Training and Research Hospital Clinical Research Ethics Committee (date/protocol: 2020/322).

\section{Statistical analysis}

Statistical analysis was performed using the Statistical Package for the Social Sciences (SPSS ${ }^{\circledR}$ version 22 ) (IBM Corp., Armonk, NY). Normal distribution of the variables was evaluated using visual (histogram and probability graphs) and analytical methods (Kolmogorov-Smirnov and Shapiro-Wilk tests). Descriptive analysis was performed, and the median and interquartile range (and frequency tables for the ordinal variables) was used to describe the non-normally distributed variables. The relationship between the mean and median values of the UPDRS and Hoehn and Yahr Scale scores obtained at the first and last visits of the patients and the side on which the clinical symptoms manifested at onset was evaluated using the MannWhitney $\mathrm{U}$ and Wilcoxon signed-rank tests. In addition, the relationship between the UPDRS and Hoehn and Yahr Scale scores obtained at the first and last visits was evaluated in relation to the age, gender, disease duration, and hand dominance of the patients using Spearman's rank-order correlation coefficient. A pvalue of $<0.05$ was considered statistically significant.

\section{Results}

There were 59 (41\%) female and 84 (59\%) male patients. The patients were aged 34-93 years (mean of $67.48 \pm$ 10.9 years). The disease duration ranged from two to 20 years (mean of $6.65 \pm 7.34$ years). The average follow-up was $3.41+0.98$ years. Seventy-six patients had right-onset PD $(53 \%)$ and 67 patients had leftonset PD (47\%) $(\mathrm{p}<0.001)$, and this finding was statistically significant $(\mathrm{p}<0.001)$. The mean age of PD onset was $61.06 \pm 10.75$ years. At both the first and last visits, the UPDRS and Hoehn and Scale scores were higher in patients with right-sided symptom onset than those in patients with left-sided symptom onset $(\mathrm{p}<$ 0.001). In addition, the differences between the scores at the first and last visits, measured using the UPDRS and the Hoehn and Yahr scale, were greater in PD patients with right-sided clinical signs at onset as compared to those with left-sided clinical symptoms at onset $(\mathrm{p}<0.002)$. Similarly, the UPDRS Part II and III values, used to evaluate motor function and measured at the first and last visits, were higher in PD patients with right-sided symptom onset as compared to those with left-sided symptom onset $(\mathrm{p}<0.001)$ (Table 1). A correlation was not found between the side of onset and disease duration using Spearman's rankorder correlation coefficient. 


\section{Cureus}

\begin{tabular}{|c|c|c|c|c|c|c|c|}
\hline \multicolumn{8}{|l|}{ ONSET SIDE } \\
\hline & \multicolumn{2}{|l|}{ Case (n) } & \multicolumn{2}{|l|}{$\mathbf{R}$} & \multicolumn{2}{|l|}{ L } & $\mathrm{p}$ \\
\hline Case (n) & \multicolumn{2}{|l|}{$143(100 \%)$} & \multicolumn{2}{|l|}{76 (53.1\%) } & \multicolumn{2}{|c|}{67 (46.9\%) } & $0.001^{\mathrm{a}}$ \\
\hline Age $^{\star} ¥$ & $67.48 \pm 10.9^{\star}$ & $69(34-93)^{*}$ & $67.64 \pm 11.27^{\star}$ & $70(34-87)^{*}$ & $67.30 \pm 10.68^{\star}$ & $69(44-93)^{¥}$ & $0.851^{\circ}$ \\
\hline Starting Age & $61.06 \pm 10.75^{*}$ & $62(31-89)^{¥}$ & $60.97 \pm 10.75^{\star}$ & $61(31-80)^{*}$ & $61.16 \pm 10.84^{\star}$ & $63(38-89)^{¥}$ & $0.916^{\odot}$ \\
\hline D Duration & $6.09 \pm 3.04^{\star}$ & $6(2-20)^{*}$ & $6.53 \pm 3.35^{\star}$ & $6(1-20)^{*}$ & $5.5 \pm 2.41^{\star}$ & $5(2-14)^{*}$ & $0.077^{\circ}$ \\
\hline Gender & & & & & & & $0.645^{\#}$ \\
\hline Female & \multicolumn{2}{|l|}{59 (41.3 \%) } & \multicolumn{2}{|l|}{30 (39.5 \%) } & \multicolumn{2}{|c|}{29 (43.3\%) } & $0.734^{\pi}$ \\
\hline Male & \multicolumn{2}{|l|}{84 (58.7 \%) } & \multicolumn{2}{|l|}{46 (60.5 \%) } & \multicolumn{2}{|c|}{38 (56.7\%) } & \\
\hline $\mathrm{T}$ & \multicolumn{2}{|l|}{$143(100 \%)$} & \multicolumn{2}{|l|}{$76(53 \%)$} & \multicolumn{2}{|c|}{$67(47 \%)$} & \\
\hline F-UPDRS§ & \multicolumn{2}{|l|}{$40^{¥}$} & \multicolumn{2}{|l|}{$44^{¥}$} & \multicolumn{2}{|l|}{$25^{¥}$} & $0.001^{\epsilon}$ \\
\hline L-UPDRS§ & \multicolumn{2}{|l|}{$51^{*}$} & \multicolumn{2}{|l|}{$56^{*}$} & \multicolumn{2}{|l|}{$30^{\neq}$} & $0.001^{\epsilon}$ \\
\hline D-FUPDRS ${ }^{\S}$ & \multicolumn{2}{|l|}{$9^{¥}$} & \multicolumn{2}{|l|}{$11^{¥}$} & \multicolumn{2}{|l|}{$5^{*}$} & $0.001^{\epsilon}$ \\
\hline F-HoenhYahr§ & \multicolumn{2}{|l|}{$2^{¥}$} & \multicolumn{2}{|l|}{$2.5^{¥}$} & \multicolumn{2}{|l|}{$2^{*}$} & $0.001^{\epsilon}$ \\
\hline L-HoenhYahr§ & \multicolumn{2}{|l|}{$3^{¥}$} & \multicolumn{2}{|l|}{$3^{*}$} & \multicolumn{2}{|l|}{$2^{*}$} & $0.001^{\epsilon}$ \\
\hline D-HoenhYahr§ & \multicolumn{2}{|l|}{0.5} & 0.5 & & 0 & & $0.002^{\epsilon}$ \\
\hline F- UPDRSpart II & $7^{*}$ & & 7 & & 4 & & $0.001^{\epsilon}$ \\
\hline F-UPDRSpart III & $22^{*}$ & & 26 & & 14 & & $0.001^{\epsilon}$ \\
\hline L-UPDRSpart II & $9^{*}$ & & 10 & & 4 & & $0.001^{\epsilon}$ \\
\hline L-UPDRSpart III & $26^{*}$ & & 28 & & 17 & & $0.001^{\epsilon}$ \\
\hline
\end{tabular}

\section{TABLE 1: Baseline characteristics and change variables according to Parkinson disease onset side}

ㄷ One-way ANOVA test used, \#Mann-Whitney U test used, *Mean Values, ¥ Median Values, D Duration: Disease Duration,

F: First, L: Last, D: Difference, §: Score, $\pi:$ Fisher exact test used, ${ }^{a}:$ T-test used, €: Wilcoxon signed-rank test used.

F- UPDRSpart II: First UPDRS - part II, F - UPDRSpart III: First UPDRS - part III, L - UPDRSpart II: Last UPDRS part II

L -UPDRSpart III: Last UPDRS part III, D - UPDRS: (difference last - first UPDRS). D-HoenhYahr: (difference last - first HoenhYahr)

ANOVA: analysis of variance

When hand dominance was evaluated, the age of PD onset was lower in left- than in right-handed patients ( $\mathrm{p}$ $<0.001)$, and PD was more common in right- than in left-handed patients $(\mathrm{p}<0.001)$. Among the left-handed patients, PD was slightly more common in women $(n=21,36 \%)$ than men $(n=9,11 \%)$. At both the first $(p<$ 0.012 ) and last visits $(\mathrm{p}<0.004)$, the UPDRS scores were higher in left- than in right-handed patients. There were no differences in D-UPDRS (difference last visits - first visits UPDRS) scores between right-handed and left-handed patients ( $p>0.05$ ). Conversely, the difference between the first visits and last visits scores was higher in right- than in left-handed patients using the Hoehn and Yahr Scale $(\mathrm{p}<0.007)$ (Table 2). A positive correlation was observed between the side of disease onset and hand dominance using Spearman's rankorder correlation coefficient. PD patients with right-sided symptom onset tended to be right-handed, and PD patients with left-sided symptom onset tended to be left-handed, using the chi-square test $(p=0.001)$. 


\section{Cureus}

\begin{tabular}{|c|c|c|c|c|c|c|c|}
\hline \multicolumn{8}{|c|}{ HAND DOMINANCE } \\
\hline & \multicolumn{2}{|l|}{ Case (n) } & \multicolumn{2}{|l|}{$\mathrm{R}$} & \multicolumn{2}{|l|}{ L } & $\mathrm{p}$ \\
\hline Case (n) & $143(100 \%)$ & & $113(79 \%)$ & & \multicolumn{2}{|c|}{$67(21 \%)$} & $0.001^{a}$ \\
\hline $\mathrm{Age}^{\star} ¥$ & $67.48 \pm 10.9^{*}$ & $69(34-93)^{¥}$ & $67.64 \pm 11.27^{\star}$ & $70(34-87)^{¥}$ & $67.30 \pm 10.68^{\star}$ & $69(44-93)^{¥}$ & $0.851^{\circ}$ \\
\hline Starting Age & $61.06 \pm 10.75^{*}$ & $62(31-89)^{*}$ & $62.72 \pm 9.81^{*}$ & $62(38-89)^{*}$ & $54.83 \pm 12.02^{\star}$ & $54(31-80)^{*}$ & $0.001^{\odot}$ \\
\hline D Duration & $6.09 \pm 3.04^{\star}$ & $6(2-20)^{*}$ & $6.26 \pm 3^{\star}$ & $6(2-20)^{*}$ & $5.4 \pm 3.17^{\star}$ & $5(2-6)^{*}$ & $0.737^{\circ}$ \\
\hline Gender & & & & & & & $0.001^{\#}$ \\
\hline Female & \multicolumn{2}{|l|}{59 (41.3 \%) } & \multicolumn{2}{|l|}{38 (64.4 \%) } & \multicolumn{2}{|c|}{21 (35.6\%) } & $0.001^{\pi}$ \\
\hline Male & \multicolumn{2}{|l|}{84 (58.7\%) } & \multicolumn{2}{|l|}{75 (89.3 \%) } & \multicolumn{2}{|c|}{$9(10.7 \%)$} & \\
\hline T & \multicolumn{2}{|l|}{$143(100 \%)$} & \multicolumn{2}{|l|}{$113(79 \%)$} & \multicolumn{2}{|c|}{30 (21 \%) } & \\
\hline F-UPDRS§ & \multicolumn{2}{|l|}{$40^{¥}$} & \multicolumn{2}{|l|}{$34^{¥}$} & \multicolumn{2}{|l|}{$45^{¥}$} & $0.012^{\epsilon}$ \\
\hline L-UPDRS\$ & \multicolumn{2}{|l|}{$51^{*}$} & \multicolumn{2}{|l|}{$47^{*}$} & \multicolumn{2}{|l|}{$59^{¥}$} & $0.004^{\epsilon}$ \\
\hline D-UPDRS§ & \multicolumn{2}{|l|}{$9^{¥}$} & \multicolumn{2}{|l|}{$8^{¥}$} & \multicolumn{2}{|l|}{$9^{*}$} & $0.212^{\epsilon}$ \\
\hline F-HoenhYahr§ & \multicolumn{2}{|l|}{$2^{¥}$} & \multicolumn{2}{|l|}{$2^{¥}$} & \multicolumn{2}{|l|}{$2.5^{*}$} & $0.476^{\epsilon}$ \\
\hline L-HoenhYahr§ & \multicolumn{2}{|l|}{$3^{¥}$} & \multicolumn{2}{|l|}{$3^{*}$} & \multicolumn{2}{|l|}{$3^{*}$} & $0.063^{\epsilon}$ \\
\hline D-HoenhYahr§ & \multicolumn{2}{|l|}{0.5} & \multicolumn{2}{|l|}{1} & \multicolumn{2}{|l|}{0.5} & $0.007^{\epsilon}$ \\
\hline
\end{tabular}

\section{TABLE 2: Baseline characteristics and change variables according to hand dominance}

๑) One-way ANOVA test used, \#Mann-Whitney U test used, *Mean Values, ¥Median Values, F: First, L: Last, D: Difference, §:

D-UPDRS: (difference last - first UPDRS) Score, a: T-test used, $\pi$ : Fisher exact-test used, $€$ : Wilcoxon signed-ranks test used,

D Duration: Disease Duration, D-HoenhYahr: (difference last - first HoenhYahr)

ANOVA: analysis of variance

PD was more common in men $(\mathrm{p}<0.001)$. At both the first $(\mathrm{p}<0.013)$ and last visits $(\mathrm{p}<0.006)$, the UPDRS scores were higher in female patients. A difference was not observed at any time in terms of the Hoehn and Yahr scale scores according to gender (Table 3). In the current study, most right-handed patients were male, and most left-handed patients were female, using the chi-square test $(\mathrm{p}=0.001)$. 


\section{Cureus}

\begin{tabular}{|c|c|c|c|c|c|c|c|}
\hline \multicolumn{8}{|c|}{ HAND DOMINANCE } \\
\hline & \multicolumn{2}{|l|}{ Case (n) } & \multicolumn{2}{|l|}{$\mathbf{R}$} & \multicolumn{2}{|l|}{$\mathrm{L}$} & $\mathrm{p}$ \\
\hline Case (n) & 143 (100\%) & & 59 (41.3 \%) & & \multicolumn{2}{|c|}{$84(58.7 \%)$} & $0.001^{\#}$ \\
\hline Age $^{\star} ¥$ & $67.48 \pm 10.9^{\star}$ & $69(34-93)^{¥}$ & $67.74 \pm 10.39^{\star}$ & $69(48-92)^{*}$ & $67.29 \pm 11.39^{*}$ & $69.5(34-93)^{*}$ & $0.811^{\odot}$ \\
\hline Starting Age & $61.06 \pm 10.75^{\star}$ & $62(31-89)^{*}$ & $61.16 \pm 10.57^{\star}$ & $60(38-89)^{¥}$ & $60.98 \pm 10.75^{\star}$ & $62.5(31-80)^{¥}$ & $0.921^{\odot}$ \\
\hline D Duration & $6.09 \pm 3.04^{*}$ & $6(2-20)^{*}$ & $5.81 \pm 2.45^{\star}$ & $5(2-12)^{*}$ & $6.23 \pm 3.43^{\star}$ & $6(2-20)$ & $0.457^{\odot}$ \\
\hline F-UPDRS§ & \multicolumn{2}{|l|}{$40^{*}$} & \multicolumn{2}{|l|}{$42^{¥}$} & \multicolumn{2}{|l|}{$33 \neq$} & $0.013^{€}$ \\
\hline L-UPDRS§ & \multicolumn{2}{|l|}{$51^{*}$} & \multicolumn{2}{|l|}{$58^{*}$} & \multicolumn{2}{|l|}{$45^{¥}$} & $0.006^{€}$ \\
\hline D-FUPDRS§ & \multicolumn{2}{|l|}{$9^{*}$} & \multicolumn{2}{|l|}{$9^{*}$} & \multicolumn{2}{|l|}{$8^{*}$} & $0.236^{\epsilon}$ \\
\hline F-HoenhYahr§ & \multicolumn{2}{|l|}{$2^{¥}$} & \multicolumn{2}{|l|}{$2.5^{*}$} & \multicolumn{2}{|l|}{$2^{¥}$} & $0.159^{€}$ \\
\hline L-HoenhYahr§ & \multicolumn{2}{|l|}{$3^{*}$} & \multicolumn{2}{|l|}{$3^{*}$} & \multicolumn{2}{|l|}{$3^{*}$} & $0.075^{\epsilon}$ \\
\hline D-HoenhYahr§ & \multicolumn{2}{|l|}{0.5} & \multicolumn{2}{|l|}{0.5} & \multicolumn{2}{|l|}{0.5} & $0.063^{\epsilon}$ \\
\hline
\end{tabular}

\section{TABLE 3: Baseline characteristics and change variables according to gender}

(ㄷ) One-way ANOVA test used, \#Mann-Whitney U test used, *Mean Values, ¥Median Values, F: First, L: Last, D: Difference, §: Score, a: T-test used, $\pi$ : Fisher exact test used, €: Wilcoxon signed-rank test used, D Duration: Disease Duration

ANOVA: analysis of variance

\section{Discussion}

Differences in brain immune function between the hemispheres have been demonstrated in animal studies [7-15]. Functional and anatomical differences between the hemispheres have also been demonstrated [1617]. Many clinical parameters have been evaluated in prognosis studies on patients with PD [18-20]. However, to the best of our knowledge, the impact of the asymmetrical presentation of clinical symptoms on the prognosis of patients with PD has not previously been evaluated, although many researchers believe that motor predominance is essential for a diagnosis of parkinsonism.

The current study investigated the effect of asymmetric symptom onset on the prognosis of PD patients. Specifically, right-sided symptom onset negatively impacted the disease prognosis, both at the onset of the disease and later. The differences between the scores, measured during the UPDRS and the Hoehn and Yahr scale at the first and last visits, were greater in PD patients with right-sided symptom onset as compared to those with left-sided symptom onset.

Higher UPDRS values were observed at both the first and last visits of patients with right-sided clinical signs at onset, especially for motor function (Part II and Part III), as compared to patients with left-sided clinical signs at onset. In addition, disease duration was higher in patients with right-sided symptom onset. Thus, right-sided symptom onset in PD patients was shown to negatively impact the functional capacity of patients, both at onset and later. These findings indicate that the nigrostriatal pathway in the left hemisphere is more sensitive to disturbance than the right hemisphere.

It is well-known that brain modulation of the immune system is asymmetric [9]. Increasing the level of interleukin-1 (IL-1) and interleukin-6 (IL-6) induces the hypothalamic-pituitary-adrenal axis [21-23]. It has been demonstrated that IL-1 is involved in brain immune modulation to a greater extent than IL-6 [15]. IL-1 and IL- 6 are produced more abundantly in the right hemisphere of the brain as compared to the left [24]. In one study, T-cell activity and natural killer (NK) cell activity decreased significantly with left hemisphere ablation, but the same effect was not seen with right hemisphere ablation [25]. This shows that much more serious functional losses may be caused by left than right hemisphere lesions due to the decrease in NK and T cells in the latter. This elucidates the greater severity of right-sided clinical manifestations, compared to left-sided clinical manifestations, at both the first and last visits in the current study, indicative of the loss of left hemisphere function.

The effect of hand dominance on motor function and its relationship with the disease has been described elsewhere $[12,26]$. In the current study, the number of right-handed patients was statistically higher than the 
number of left-handed patients. In most right-handed patients, the left hemisphere is the dominant hemisphere [27-28]. Therefore, in the current study, it was unsurprising that PD was more common in righthanded patients since the left hemisphere is more vulnerable to disturbance than the right hemisphere. However, Parkinson's disease starts at a much earlier age in left-handed patients, and the neurological disability is also severe. The dominant hemisphere is primarily the right hemisphere in left-handed people; however, the left hemisphere can be dominant in $15 \%-20 \%$ of cases [27-28]. The dominant hemisphere and immunological differences in left-handed people may be more complicated than what has been established currently. In the current study, most left-handed patients were also found to be women. The difference between the first and last visits scores was higher in right- than in left-handed patients using the Hoehn and Yahr scale.

A relationship was not found between hand dominance, disease duration, and the age of PD patients. In many diseases, the dominant hemisphere and hand use differ vastly from the healthy population. For example, Lewin et al. reported that Down's syndrome, epilepsy, and autism patients were characterized by a significant increase in left-handedness and hand use complexity [29]. Pain was frequently experienced on the left side of the head in a study that evaluated migraines in left-handed patients [30]. In the current study, PD was more common in male and right-handed patients, although women and left-handed patients had greater neurological disability assessed using UPDRS scores. Although PD starts worse in left-handed people in terms of neurological disability than right-handed people, the progression rate of the disease in righthanded people (D-HoenhYahr) is higher than in left-handed people. Thus, gender and hand dominance could be factors that affect central asymmetric immune function in PD patients, which is not the case in the healthy population. Although the current study was retrospective and the number of patients included was relatively limited, it is notable that it is the first study to have evaluated the effect of lateralization on the prognosis of patients with $\mathrm{PD}$.

\section{Conclusions}

The right-sided onset clinical findings were negative prognostic factors in patients with Parkinson's disease. We believe that the presence of right-sided clinical findings at the onset is an important guide in the regulation of follow-up and prognosis and the treatment of these patients.

\section{Additional Information \\ Disclosures}

Human subjects: Consent was obtained or waived by all participants in this study. Karabuk University Training and Research Hospital Clinical Researches Ethics Committee issued approval (date/protocol: 2020/322). Animal subjects: All authors have confirmed that this study did not involve animal subjects or tissue. Conflicts of interest: In compliance with the ICMJE uniform disclosure form, all authors declare the following: Payment/services info: All authors have declared that no financial support was received from any organization for the submitted work. Financial relationships: All authors have declared that they have no financial relationships at present or within the previous three years with any organizations that might have an interest in the submitted work. Other relationships: All authors have declared that there are no other relationships or activities that could appear to have influenced the submitted work.

\section{References}

1. Djaldetti R, Ziv I, Melamed E: The mystery of motor asymmetry in Parkinson's disease . Lancet Neurol. 2006, 5:796-802. 10.1016/S1474-4422(06)70549-X

2. Kempster PA, Gibb WR, Stern GM, Lees AJ: A symmetry of substantia nigra neuronal loss in Parkinson's disease and its relevance to the mechanism of levodopa related motor fluctuations. J Neurol Neurosurg Psychiatry. 1989, 52:72-76. 10.1136/jnnp.52.1.72

3. McRitchie DA, Halliday GM, Cartwright H: Quantitative analysis of the variability of substantia nigra pigmented cell clusters in the human. Neuroscience. 1995, 68:539-551. 10.1016/0306-4522(95)00163-d

4. Halliday GM, Li YW, Blumbergs PC, et al.: Neuropathology of immunohistochemically identified brainstem neurons in Parkinson's disease. Ann Neurol. 1990, 27:373-385. 10.1002/ana.410270405

5. Ma SY, Collan Y, Roytta M, Rinne JO, Rinne UK: Cell counts in the substantia nigra: a comparison of single section counts and disector counts in patients with Parkinson's disease and in controls. Neuropathol Appl Neurobiol. 1995, 21:10-17. 10.1111/j.1365-2990.1995.tb01023.x

6. Knossalla F, Kohl Z, Winkler J, et al.: High-resolution diffusion tensor-imaging indicates asymmetric microstructural disorganization within substantia nigra in early Parkinson's disease. J Clin Neurosci. 2018, 50:199-202. 10.1016/j.jocn.2018.01.023

7. Neveu PJ, Betancur C, Barnéoud P, Vitiello S, Le Moal M: Functional brain asymmetry and lymphocyte proliferation in female mice: effects of right and left cortical ablation. Brain Res. 1991, 31:125-128. 10.1016/0006-8993(91)90414-q

8. Gontova IA, Abramov VV, Kozlov VA: Asymmetry of delayed type hypersensitivity reaction in mice. Bull Exp Biol Med. 2003, 135:67-69. 10.1023/a:1023454114499

9. Gontova IA, Abramov VV, Kozlov VA: The role of asymmetry of nervous and immune systems in the formation of cellular immunity of (CBaxC57Bl/6) F1 mice. Neuroimmunomodulation. 2004, 11:385-391. 10.1159/000080149

10. Dane S, Borekci B, Kadanali S: Right-sided lateralisation of ovarian cancer and right bias asymmetry for involved pelvic lymph nodes by ovarian cancer cells. Laterality. 2008, 13:393-402. 
$10.1080 / 13576500801957636$

11. Yoruk O, Karasen M, Timur H, Erdem T, Dane S, Tan U: Lateralizations of head-neck cancers are not associated with peripheral asymmetry of cell-mediated immunity. Int J Neurosci. 2009, 119:815-820. 10.1080/00207450802329639

12. Kramer MA, Albrecht S, Miller RA: Handedness and the laterality of breast cancer in women . Nurs Res. 1985, 34:333-337.

13. Varoglu AO, Balkuv $\mathrm{E}$ : Right-sided clinical findings are worse prognostic factor in multiple sclerosis patients?. Neurosciences. 2020, 25:97-103. 10.17712/nsj.2020.2.20190111

14. Goetz GC, Fahn S, Martinez-Martin P, et al.: Movement Disorder Society-sponsored revision of the Unified Parkinson's Disease Rating Scale (MDS-UPDRS): process, format, and clinimetric testing plan. Mov Disord. 2007, 22:41-47. 10.1002/mds.21198

15. Shen YQ, Hébert G, Moze E, Li KS, Neveu PJ: Asymmetrical distribution of brain interleukin-6 depends on lateralization in mice. Neuroimmunomodulation. 2005, 12:189-194. 10.1159/000084852

16. Sadeghi L, Rizvanov AA, Salafutdinov II, Dabirmanesh B, Sayyah M, Fathollahi Y, Khajeh K: Hippocampal asymmetry: differences in the left and right hippocampus proteome in the rat model of temporal lobe epilepsy. J Proteomics. 2017, 10:22-29. 10.1016/j.jprot.2016.11.023

17. Vingerhoets G, Li X, Hou L, et al.: Brain structural and functional asymmetry in human situs inversus totalis. Brain Struct Funct. 2018, 223:1937-1952. 10.1007/s00429-017-1598-5

18. Aarsland D: Cognitive impairment in Parkinson's disease and dementia with Lewy bodies . Parkinsonism Relat Disord. 2016, 22:S144-148. 10.1016/j.parkreldis.2015.09.034

19. Park K, Oeda T, Kohsaka M, Tomita S, Umemura A, Sawada H: Low body mass index and life prognosis in Parkinson's disease. Parkinsonism Relat Disord. 2018, 55:81-85. 10.1016/j.parkreldis.2018.05.011

20. Hussain M, Kumar P, Khan S, Gordon DK, Khan S: Similarities between depression and neurodegenerative diseases: pathophysiology, challenges in diagnosis and treatment options. Cureus. 2020, 21:e11613. 10.7759/cureus.11613

21. Chesnokova V, Melmed S: Minireview: neuroimmuno-endocrine modulation of the hypothalamic-pituitaryadrenal (HPA) axis by gp130 signaling molecules. Endocrinology. 2002, 143:1571-1574. 10.1210/endo.143.5.8861

22. Gerendai I, Halász B: Neuroendocrine asymmetry. Front Neuroendocrinol. 1997, 18:354-381. 10.1006/frne.1997.0154

23. Delrue C, Deleplanque B, Rouge-Pont F, Vitiello S, Neveu PJ: Brain monoaminergic, neuroendocrine, and immune responses to an immune challenge in relation to brain and behavioral lateralization. Brain Behav Immun. 1994, 8:137-152. 10.1006/brbi.1994.1013

24. Fu QL, Shen YQ, Gao MX, Dong J, Neveu PJ, Li KS: Brain interleukin asymmetries and paw preference in mice. Neuroscience. 2003, 116:639-647. 10.1016/s0306-4522(02)00746-7

25. Bardos P, Degenne D, Lebranchu Y, Bizière K, Renoux G: Neocortical lateralization of NK activity in mice. Scand J Immunol. 1981, 13:609-611. 10.1111/j.1365-3083.1981.tb00176.x

26. Tan U: Left-sided lateralization of immune disorders in a left-handed female subject . Int J Neurosci. 1989, 48:367-368. 10.3109/00207458909002182

27. Metzig E, Rosenberg S, Ast M: Lateral asymmetry in patients with nervous and mental disease. A preliminary study. Neuropsychobiology. 1975, 1:197-202. 10.1159/000117493

28. Muraleedharan A, Ragavan S, Devi R: Are footedness and lateral postures better predictors of hemispheric dominance than handedness: a cross-sectional questionnaire-based clinical and pedigree study. J Neurosci Rural Pract. 2020, 11:130-134. 10.1055/s-0039-3400699

29. Lewin J, Kohen D, Mathew G: Handedness in mental handicap: investigation into populations of Down's syndrome, epilepsy and autism. Br J Psychiatry. 1993, 163:674-676. 10.1192/bjp.163.5.674

30. Geschwind N, Behan P: Left-handedness: association with immune disease, migraine, and developmental learning disorder. Proc Natl Acad Sci U S A. 1982, 79:5097-5100. 10.1073/pnas.79.16.5097 